\title{
YAP overexpression promotes the epithelial-mesenchymal transition and chemoresistance in pancreatic cancer cells
}

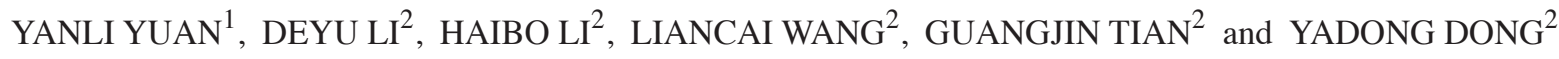 \\ ${ }^{1}$ Children's Hospital of Zhengzhou, Zhengzhou, Henan 450008; \\ ${ }^{2}$ Henan Provincial People's Hospital, Zhengzhou, Henan 450003, P.R. China
}

Received January 9, 2014; Accepted October 27, 2015

DOI: $10.3892 / \mathrm{mmr} .2015 .4550$

\begin{abstract}
The expression of Yes-associated protein (YAP) has been reported to be dysregulated in pancreatic cancer. However, its contributions to tumor formation and progression remain to be elucidated. The present study demonstrated that YAP overexpression promoted the epithelial-mesenchymal transition (EMT) in a manner associated with pancreatic cancer invasion in vitro. RNA interference-mediated silencing of YAP attenuated cell invasion in vitro. Mechanistically, the present study demonstrated that YAP overexpression fosters pancreatic cancer progression by inducing the EMT in pancreatic cancer cells by activating the AKT cascade, which can counteract the effect of gemcitabine. These data suggested that the YAP acts synergistically to promote pancreatic cancer progression by hyperactivation of AKT signaling. The present study revealed YAP as a potential therapeutic target for pancreatic cancer and a biomarker for predicting gemcitabine treatment response.
\end{abstract}

\section{Introduction}

Pancreatic cancer is the fourth leading cause of cancer-associated mortality worldwide (1). This cancer type is characterized by early metastasis, and pronounced resistance to chemotherapy and radiation (2-4). Although systemic treatment, including gemcitabine and erlotinib, has been used for advanced pancreatic cancer, the effect of current chemotherapy is only a small survival advantage (5-7). Therefore, identification of novel therapeutic targets and approaches are required against pancreatic cancer to improve patient prognosis.

Correspondence to: Professor Yadong Dong, Henan Provincial People's Hospital, 7 Weiwu Road, Zhengzhou, Henan 450003, P.R. China

E-mail: dongyadong_hn@163.com

Abbreviations: EMT, epithelial-mesenchymal transition; YAP, Yes-associated protein

Key words: pancreatic cancer, EMT, YAP, chemoresistance
Yes-associated protein (YAP) overexpression has been reported for several human tumor entities, including prostate, ovarian, colon, liver, lung and pancreatic cancer (8-10). Several previous studies have suggested that dysregulation of the YAP cascade may be critically involved in the development of several tumor types (11-15). In addition, the expression of YAP correlates with poor prognosis in different cancer types, including colorectal, esophageal, gastric, hepatocellular, lung and ovarian (9,16-20). The Hippo pathway is important in tumorigenesis (21) and YAP was first noted as an oncogene from a previous study of the Hippo/YAP pathway, which regulates the balance between cell proliferation and apoptosis (22). It also has been confirmed in a previous study that YAP functions as a critical transcriptional switch downstream of the oncogenic KRAS-mitogen-activated protein kinase pathway in pancreatic cancer (15).

The present study revealed that YAP overexpression promoted the epithelial-mesenchymal transition (EMT) of pancreatic cancer cells and increased drug resistance. The role of YAP on the sensitivity of pancreatic cancer cells to gemcitabine was investigated and the present study explored the mechanisms, which may mediate such an effect. The findings of the present study suggested that YAP induces the EMT and regulates the sensitivity of pancreatic cancer cells to gemcitabine by activating AKT and raises the possibility that YAP may be a promising target to improve the efficacy of therapy for pancreatic cancer.

\section{Materials and methods}

Cells and clinical samples. The pancreatic cancer cell lines, PANC-1, MIA PaCa-2, BxPC-3, Capan-1, T3M4 and colo357, were purchased from American Type Culture Collection (Rockville, MD, USA). The BxPC-3 cells were grown in RPMI-1640 medium, containing 10\% fetal bovine serum (FBS) and penicillin/streptomycin (all Gibco; Thermo Fisher Scientific, Inc., Waltham, MA, USA). The PANC-1, MIA PaCa-2, T3M4 and colo357 cells were cultured in Dulbecco's modified Eagle's medium (Gibco; Thermo Fisher Scientific, Inc.), containing 10\% FBS and penicillin/streptomycin.

Fresh-frozen specimens of human normal pancreatic tissues and primary pancreatic cancer tissues were obtained along with written informed consent and pathology reports from the Henan Provincial People's Hospital (Henan, China), and were 


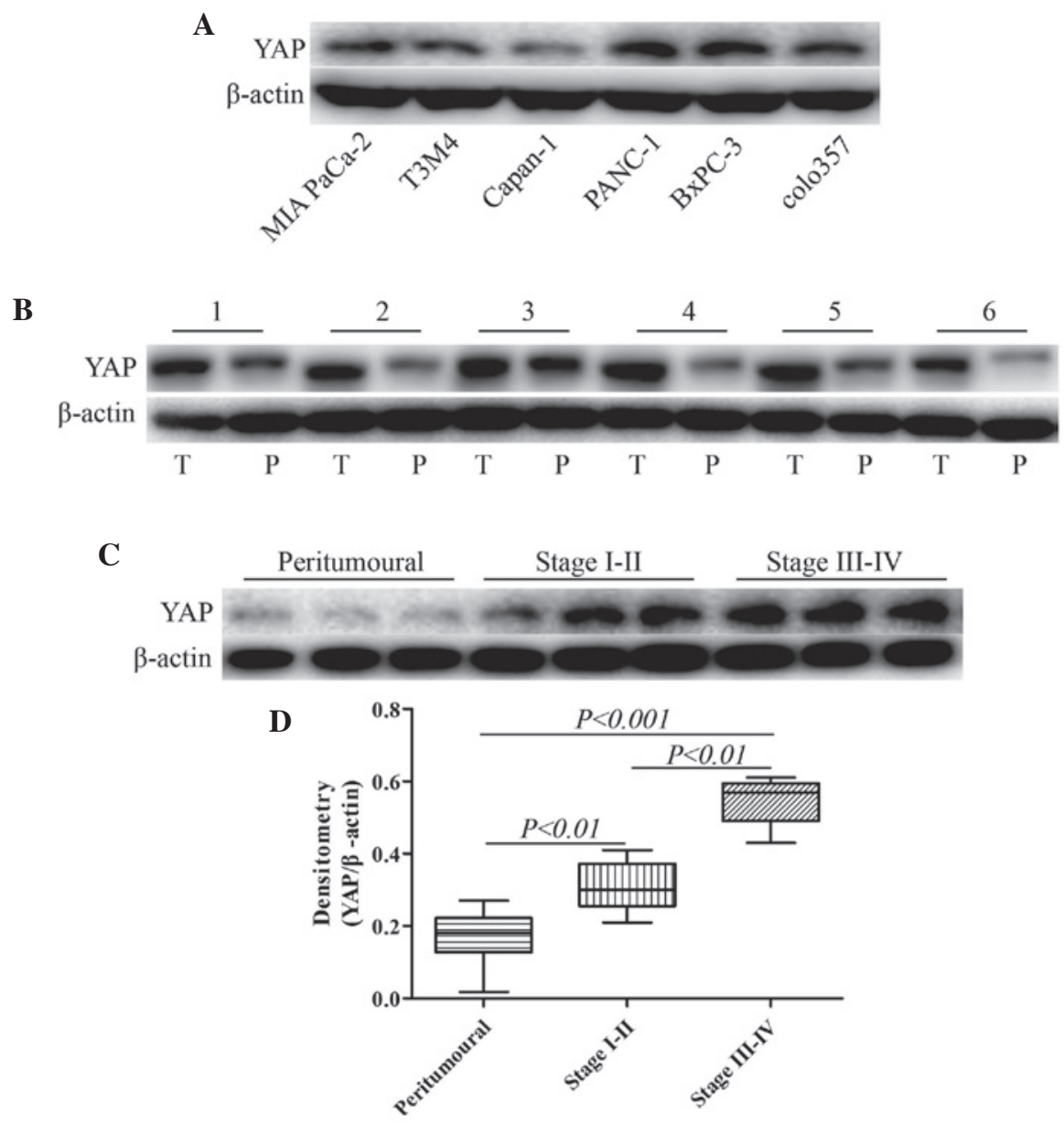

Figure 1. YAP is upregulated in human pancreatic cancer. The relative protein expression levels of YAP was determined in (A) different pancreatic cancer cell lines, (B) tumor and peritumoral tissues from patients with pancreatic cancer and (C) in fresh-frozen specimens from pancreatic cancer and matched peritumoral tissues by western blot analysis. (D) Densitometric quantitation of the western blots is also shown for the fresh-frozen specimens. $\beta$-actin was used as a loading control. T, tumor; P, peritumoral; YAP, YES-associated protein.

used for reverse transcription-quantitative polymerase chain reaction (RT-qPCR) and western blotting. Sample collection was performed following approval from the institutional Ethics Review Committee of the Henan Provincial People's Hospital. No patient had undergone chemotherapy prior to surgery.

Western blotting. The cells were lysed in cell lysis buffer for western and IP (Beyotime Institute of Biotechnology, Haimen, China) to obtain the total cellular protein. The protein concentrations were determined using an Enhanced BCA protein assay kit (Beyotime Institute of Biotechnology) and were subsequently boiled for $10 \mathrm{~min}$ at $100^{\circ} \mathrm{C}$. The protein samples $(2 \mu \mathrm{g} / \mu \mathrm{l} ; 30 \mu \mathrm{g}$ ) were separated by $12 \%$ SDS-PAGE (Beyotime Institute of Biotechnology) and were subsequently transferred onto polyvinylidene difluoride membranes (Bio-Rad Laboratories, Inc., Hercules, CA, USA). The membranes were rinsed in Tris-buffered saline, containing $0.1 \%$ Tween-20 and blocked with $5 \%$ bovine serum albumin (Beyotime Institute of Biotechnology) for $2 \mathrm{~h}$ at room temperature. Following blocking, the membranes were incubated with the following primary antibodies at $4{ }^{\circ} \mathrm{C}$ overnight: Mouse monoclonal anti-YAP (1:500; cat.no. sc-376830; Santa Cruz Biotechnology, Inc., Dallas, TX, USA), rabbit monoclonal anti-E-cadherin (1:1,000; cat. no. 3195; Cell Signaling Technology, Inc.,
Danvers, MA, USA), rabbit monoclonal anti-N-cadherin (1:1,000; cat. no. 13116; Cell Signaling Technology, Inc.), rabbit monoclonal anti-snail (1:1,000; cat. no. 3879; Cell Signaling Technology, Inc.), rabbit monoclonal anti-phosphorylated (p)-AKT (1:1,000; cat. no. 4060; Cell Signaling Technology, Inc.) and mouse monoclonal anti- $\beta$-actin (1:1,000; cat. no. sc-130065; Santa Cruz Biotechnology, Inc.). The membranes were rinsed in phosphate-buffered saline containing $0.1 \%$ Tween-20 and incubated with horseradish peroxidase-conjugated goat anti-mouse (cat. no. A0216) and goat anti-rabbit (cat. no. A0208) secondary antibodies (1:1,000; Beyotime Institute of Biotechnology) for $2 \mathrm{~h}$ at room temperature. Following washing, the proteins were detected using enhanced chemiluminescence (Beyotime Institute of Biotechnology).

Ipatasertib-induced AKT inhibition. The cells were treated with the AKT inhibitor ipatasertib $(0.5 \mu \mathrm{M}$; Anpei, Nanjing, China) for $24 \mathrm{~h}$. Subsequently, cell lysates were prepared and western blotting was performed.

Transwell migration and invasion assay. Cell migration and invasion were investigated using a Transwell migration assay and a matrigel invasion assay $(8 \mu \mathrm{m}$ pore size; BD Falcon, 
A

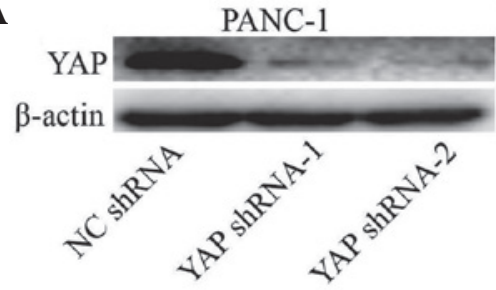

B

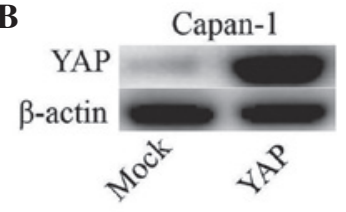

\section{C}
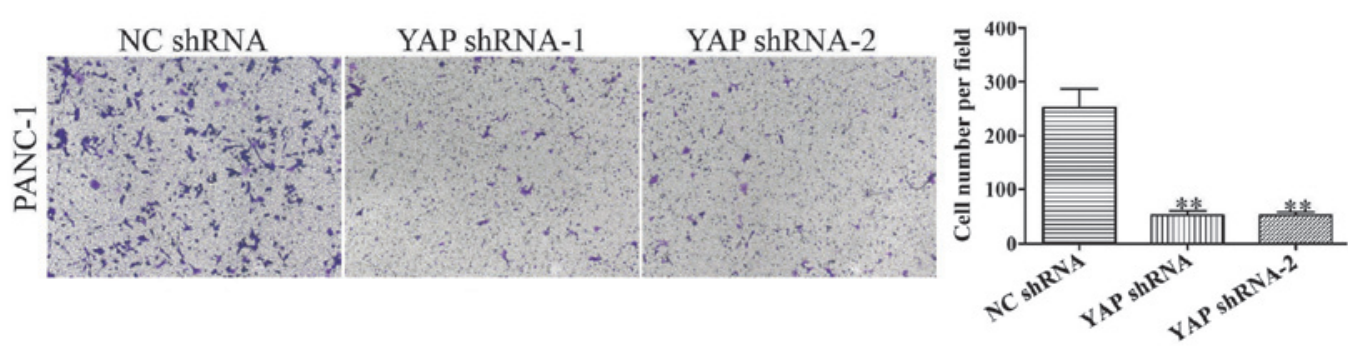

D
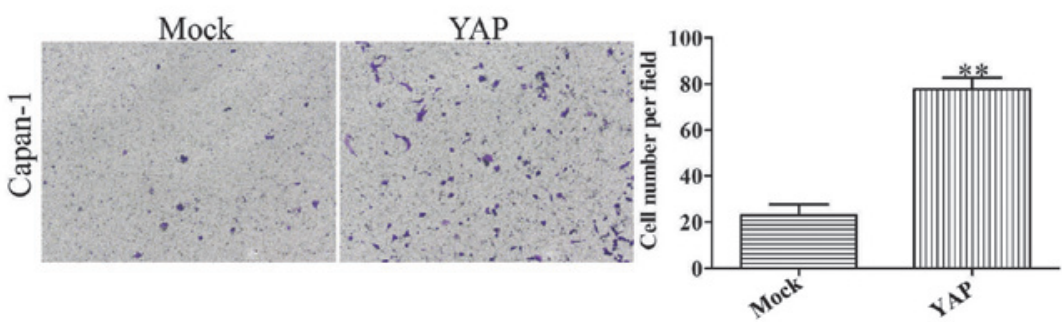

Figure 2. Overexpression of YAP promotes pancreatic cancer invasion in vitro. (A and B) The expression of YAP was determined in pancreatic cancer cells modified by shRNA and cDNA transfection. (C and D) The invasion of the cancer cells was measured by Transwell assays. The data are expressed as the mean \pm standard deviation $\left(\mathrm{n}=3 ;{ }^{* *} \mathrm{P}<0.01\right)$. YAP, YES-associated protein; sh, short hairpin.

San Jose, CA, USA), as described previously (23). Briefly, for the Transwell migration assay, $5 \times 10^{4}$ cells were suspended in $200 \mu \mathrm{l}$ serum-free DMEM and placed in the cell culture insert of a Transwell plate, and warmed culture medium supplemented with $10 \%$ FBS was placed in the well. Cells in serum-free DMEM were seeded in the upper chamber and medium containing FBS was seeded in the lower chamber. For the matrigel invasion assay, $2 \times 10^{5}$ cells were suspended in $200 \mu \mathrm{l}$ DMEM without FBS and then placed in the cell culture insert precoated with $1 \mu \mathrm{g} / \mu 1$ Matrigel (BD Biosciences). Warmed culture medium containing $10 \%$ FBS was added to the well. The cells were cultured for $12 \mathrm{~h}$ at $37^{\circ} \mathrm{C}$ in an atmosphere containing $5 \% \mathrm{CO}_{2}$ and were fixed in $4 \%$ paraformaldehyde and stained with $0.1 \%$ crystal violet (Sigma-Aldrich). The number of migrated cells in five randomly selected fields was counted under a light microscope (magnification, x100; Olympus, Tokyo, Japan).

Drug sensitivity assay. To determine drug sensitivity, the cells were seeded into 96 -well plates at a density of $2 \times 10^{3}$ cells/well. Following incubation for $24 \mathrm{~h}$, the cells were placed in complete medium, containing different concentrations of gemcitabine (0.2, 1, 5, 25, $125 \mu \mathrm{M}$; Jiangsu Hansoh Pharmaceutical Co., Ltd., Lianyugang, China). Following incubation for a further $72 \mathrm{~h}$, the sensitivity of the cells to gemcitabine was measured using a cell counting kit-8 (Dojindo Molecular Technologies, Inc., Kumamoto, Japan).

Lentivirus production and transduction of target cells. The YAP and YAP short hairpin (sh)RNA expression lentivirus were purchased from Shanghai GeneChem Co., Ltd. (Shanghai, China) and the target shRNA sequences were as follows: 5'-CTCAGGATGGAGAAATTTA-3' and 5'-CGT GCCCCAGACCGTGCCC-3'. The lentiviral vector was transfected into cells, as described previously (24), cancer cells were infected with lentivirus plus $6 \mu \mathrm{g} / \mathrm{ml}$ polybrene (Sigma-Aldrich) for $24 \mathrm{~h}$, and transfection was confirmed by immunoblotting.

Statistical analysis. Statistical analysis was performed using SPSS 12.0 software (SPSS, Inc., Chicago, IL, USA). The data are expressed as the mean \pm standard deviation. The data were examined using analysis of variance and the least significant differences method for multisample comparisons, or Student's t-test for two-sample comparisons. Kaplan-Meier curves were plotted to assess the effects of YAP expression on the progression-free survival. Survival curves were compared using the log-rank test. $\mathrm{P}<0.05$ was considered to indicate a statistically significant difference.

\section{Results}

YAP expression is upregulated in pancreatic cancer tissues and this expression correlates with cancer progression. To explore the role of YAP in pancreatic cancer progression, the expression of YAP was assessed in various human pancreatic cancer cell lines, pancreatic cancer and matched peritumoral tissues. The expression of YAP in 30 pancreatic cancer and matched peritumoral tissues was analyzed by western blotting. Compared with the peritumoral samples, 

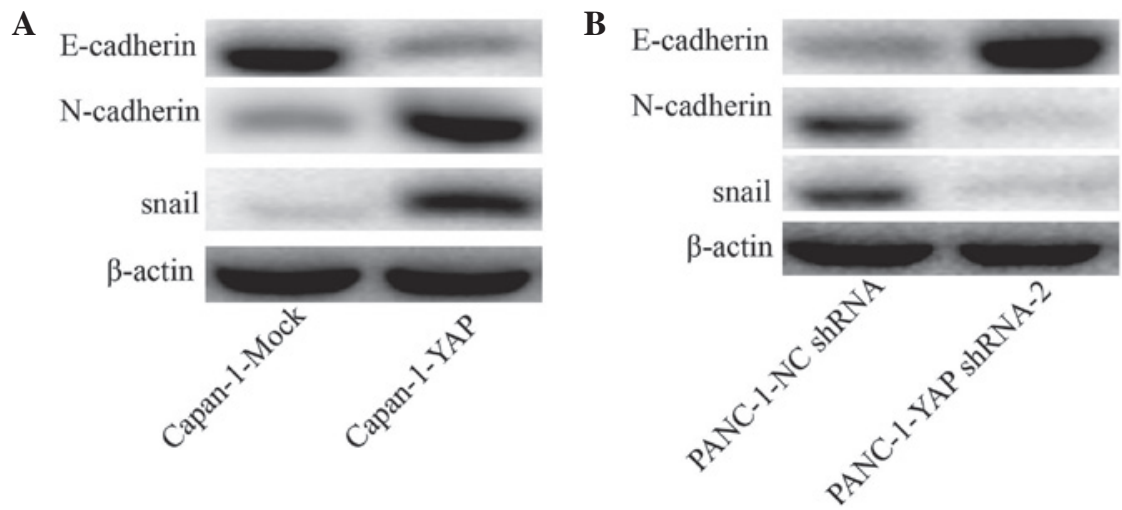

Figure 3. YAP overexpression mediates the EMT of the pancreatic cancer cells. (A and B) EMT markers were observed between high and low expression YAP cells (Capan-1-YAP, vs. Capan-1-Mock and PANC-1-YAP shRNA, vs. PANC-1-NC shRNA). YAP, YES-associated protein; EMT, epithelial-mesenchymal transition.

A

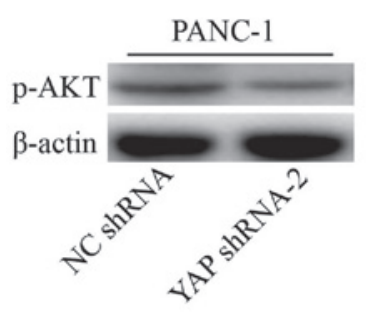

B

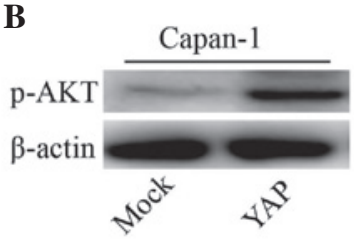

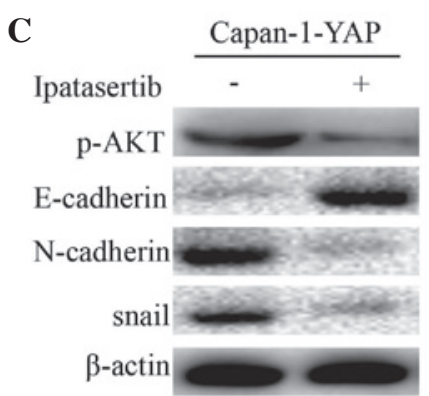

Figure 4. YAP-mediated pancreatic cancer cell EMT by the hyperactivation of AKT. (A) The expression of p-AKT is downregulated by the knockdown of YAP in pancreatic cancer cells. (B) The forced expression of YAP upregulated the expression of p-AKT in pancreatic cancer cells. (C) The AKT specific inhibitor, ipatasertib, reversed the EMT conferred by YAP overexpression in Capan-1 cells. YAP; YES-associated protein; EMT, epithelial-mesenchymal transition; p-, phosphorylated; sh, short hairpin.

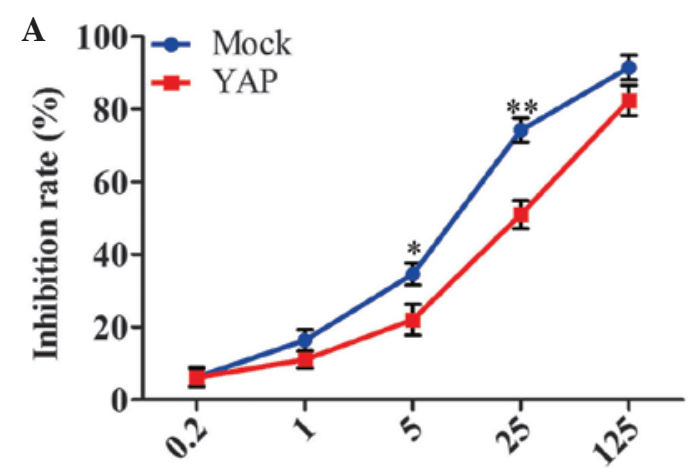

Gemcitabine concentration $(\mu \mathrm{M})$

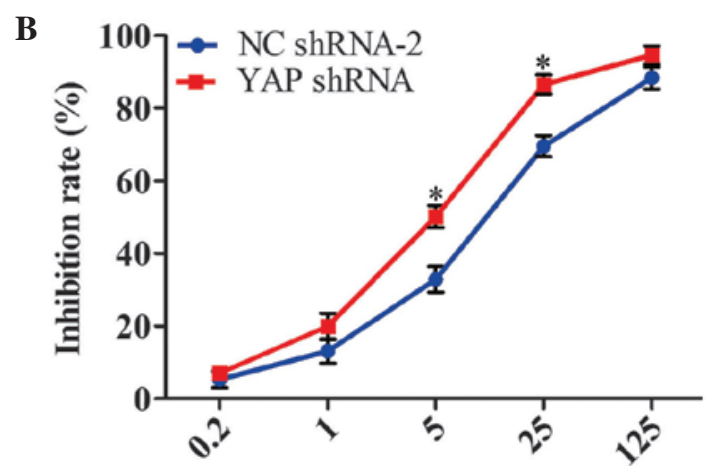

Gemcitabine concentration $(\mu \mathrm{M})$

Figure 5. YAP induces the resistance to gemcitabine of pancreatic cancer cells. (A) Overexpression of YAP in Capan-1 cells reduced the sensitivity of the cells to gemcitabine. (B) Knockdown of the expression of YAP in PANC-1 cells increased the sensitivity of the cells to gemcitabine. The data are expressed as the mean \pm standard deviation $\left(n=3 ;{ }^{*} \mathrm{P}<0.05\right.$ and $\left.{ }^{* *} \mathrm{P}<0.01\right)$. YAP, YES-associated protein.

semi-quantitative analysis revealed that the protein expression levels of YAP were markedly higher in the cancer tissues (Fig. 1A and B). By contrast, in normal pancreatic tissues, little YAP expression was observed. YAP expression in early-stage (I-II) and advanced-stage (III-IV) pancreatic cancer tissues was significantly higher compared with that in normal pancreatic tissues $(\mathrm{P}<0.01)$. In addition, YAP expression in advanced-stage (III-IV) was significantly higher compared with in early-stage (I-II) pancreatic cancer tissues $(\mathrm{P}<0.01$; Fig. 1C).
YAP is involved in pancreatic cancer cell invasion in vitro. To elucidate the role of YAP in pancreatic cancer progression, YAP shRNAs were used to reduce the expression of YAP in the human PANC-1 pancreatic cancer cells, which exhibit a high level of YAP protein expression (Fig. 1A). YAP shRNAs significantly reduced the expression of YAP, as well as the invasion of PANC-1 cells ( $\mathrm{P}<0.01$; Fig. $2 \mathrm{~A}$ and $\mathrm{C})$. To further evaluate whether YAP upregulation promoted tumor invasion, lentivirus-mediated delivery of YAP cDNA was used to increase the expression of YAP in human Capan-1 pancreatic 
cancer cells, which exhibit low protein expression of YAP (Fig. 1A). Upregulation of the regulation of YAP was observed in YAP infectants (Fig. 2B). YAP upregulation significantly increased the invasion ability of Capan-1 cells compared with the mock control (Fig. 2D). Collectively, the data from the in vitro assays revealed that YAP significantly contributed to tumor invasion of pancreatic cancer.

YAP regulates the EMT phenotypes in pancreatic cancer cells. Based on the association between the expression of YAP and the invasion of pancreatic cancer in vitro, and since that the EMT is considered a striking feature of most cancer types and has a vital role in cancer migration and invasion, the present study compared the expression of epithelial and mesenchymal markers, as well as other molecules thought to induce EMT in cancer cells. As shown in Fig. 3A, Capan-1-YAP cells expressed a lower level of the epithelial gene, E-cadherin, compared with Capan-1-Mock cells. The mesenchymal genes, snail and N-cadherin, were significantly upregulated in Capan-1-YAP cells compared with Capan-1-Mock cells. Notably, the level of E-cadherin was higher in PANC-1-YAP shRNA compared with PANC-1-NC shRNA cells, while mesenchymal-associated genes, snail and $\mathrm{N}$-cadherin, were downregulated in PANC-1-YAP shRNA cells (Fig. 3B).

YAP-mediated EMT occurs through the activation of the AKT signaling pathway. It has been previous confirmed that the induction of the EMT may be an important mechanism of constitutive AKT signaling activation in various cancer types. To further understand whether the YAP-mediated EMT process in pancreatic cancer cells was dependent on the activation of the AKT pathway, western blotting analysis was performed to assess the activation of the components of the AKT pathway in YAP-knockdown or -overexpressing pancreatic cancer cells. The results indicated that shRNA-mediated YAP downregulation in PANC-1 cells markedly reduced the expression of $\mathrm{p}-\mathrm{AKT}$ (Fig. 4A), whereas YAP overexpression in Capan-1 cells increased the expression of p-AKT (Fig. 4B). Finally, the present study analyzed the effect of ipatasertib-mediated p-AKT inhibition on the expression levels of E-cadherin, $\mathrm{N}$-cadherin and snail in pancreatic cancer cells. Notably, the expression levels of $\mathrm{N}$-cadherin and snail were markedly downregulated, while the expression of E-cadherin was markedly upregulated in cancer cells treated with ipatasertib (Fig. 4C). These results indicated that YAP induced the EMT by way of hyperactivation of AKT signaling in pancreatic cancer cells.

YAP modulates the chemoresistance of human pancreatic cancer cells. The present study further investigated whether increasing or inhibiting the expression of YAP modulated the sensitivity of pancreatic cancer cells to gemcitabine, which is currently used as the first line treatment for pancreatic cancer. Following exogenous expression of YAP in Capan-1 cells, the cells were treated with a series of concentrations of gemcitabine $(0.2,5,25$ and $125 \mu \mathrm{M})$. The effect of YAP on the chemoresistance of Capan-1 cells is shown in Fig. 5A. The half-maximal inhibitory concentrations $\left(\mathrm{IC}_{50}\right)$ of gemcitabine on Capan-1-Mock and Capan-1-YAP cells were $8.52 \pm 1.88$ and $21.56 \pm 3.03 \mu \mathrm{M}$, respectively $(\mathrm{P}<0.05)$. These results indicated that the introduction of YAP notably reduced the chemosensitivity of Capan-1 cells to gemcitabine. In addition, the inhibition of PANC-1 cell growth with gemcitabine was significantly increased by transfection with YAP shRNA. The $\mathrm{IC}_{50}$ values of gemcitabine on PANC-1-NC shRNA-2 and PANC-1-YAP shRNA cells were $14.22 \pm 1.45$ and $4.88 \pm 0.61$ $\mu \mathrm{M}$, respectively $(\mathrm{P}<0.05$; Fig. $5 \mathrm{~B})$.

\section{Discussion}

YAP is a multifunctional molecule that regulates cell survival, proliferation, migration and differentiation in several human cancer types (25-27). In the present study, based on depletion and overexpression experiments in vitro, it was revealed that YAP has a crucial role in regulating pancreatic cancer invasion and chemoresistance to gemcitabine.

Increasing evidence from experimental and clinical studies suggest that the EMT is important in tumor invasion, migration and metastasis (28-30). The EMT is observed in a series of cancer cells undergoing phenotypic conversion for invasion and metastasis, and is characterized by the gain of mesenchymal markers, including snail and N-cadherin, and the loss of epithelial cell junction proteins, including E-cadherin (31). The present study reported that cells, which express high levels of YAP, expressed high levels of snail, N-cadherin and low levels of E-cadherin, suggesting that YAP may be a potent inducer of the EMT, which may result in increased invasion and migration of pancreatic cancer cells. Therefore, the YAP-induced EMT may be a major contributing factor to the invasion of pancreatic cancer cells.

In models of chemotherapy resistant cancer types, EMT gene signatures have been hypothesized to be involved in the presence of chemotherapy resistance, and regulation of EMT transcriptional regulators modulates resistance to chemotherapeutic agents $(32,33)$. Emerging evidence suggests that the EMT is involved in cancer progression, and targeting the EMT can reverse the resistance of antitumor drugs (34). Furthermore, it was also confirmed in previous studies that hyperactivation of AKT signaling is involved in the chemoresistance of pancreatic cancer $(35,36)$. The present findings demonstrated that the gemcitabine resistance of pancreatic cancer was due, in part, to the presence of YAP. YAP significantly increased the activation of AKT, which can enhance gemcitabine resistance in pancreatic cancer.

In conclusion, the results of the present study revealed that YAP is expressed in pancreatic cancer tissues and is positively correlated with tumor progression. The overexpression of YAP may contribute to the invasiveness of pancreatic cancer cells. Additionally, the present study provided evidence of a molecular and phenotypic association between the YAP-induced EMT phenotype and gemcitabine-resistance of pancreatic cancer cells. YAP expression reduces the sensitivity to gemcitabine in pancreatic cancer cells. Taken together, YAP is important for the pathogenesis pancreatic cancer and may be a biomarker for predicting response to gemcitabine treatment.

\section{References}

1. Jemal A, Siegel R, Xu J and Ward E: Cancer statistics, 2010. CA Cancer J Clin 60: 277-300, 2010. 
2. Maheshwari V and Moser AJ: Current management of locally advanced pancreatic cancer. Nat Clin Pract Gastroenterol Hepatol 2: 356-364, 2005

3. Wang Z, Li Y, Ahmad A, Banerjee S, Azmi AS, Kong D and Sarkar FH: Pancreatic cancer: Understanding and overcoming chemoresistance. Nat Rev Gastroenterol Hepatol 8: 27-33, 2011.

4. Goodman KA and Hajj C: Role of radiation therapy in the management of pancreatic cancer. J Surg Oncol 107: 86-96, 2013.

5. Mahalingam D, Kelly KR, Swords RT, Carew J, Nawrocki ST and Giles FJ: Emerging drugs in the treatment of pancreatic cancer. Expert Opin Emerg Drugs 14: 311-328, 2009.

6. Cunningham D, Chau I, Stocken DD, Valle JW, Smith D, Steward W, Harper PG, Dunn J, Tudur-Smith C, West J, et al: Phase III randomized comparison of gemcitabine versus gemcitabine plus capecitabine in patients with advanced pancreatic cancer. J Clin Oncol 27: 5513-5518, 2009.

7. Herrmann R, Bodoky G, Ruhstaller T, Glimelius B, Bajetta E, Schüller J, Saletti P, Bauer J, Figer A, Pestalozzi B, et al: Gemcitabine plus capecitabine compared with gemcitabine alone in advanced pancreatic cancer: A randomized, multicenter, phase III trial of the Swiss group for clinical cancer research and the central European cooperative oncology group. J Clin Oncol 25: 2212-2217, 2007.

8. Steinhardt AA, Gayyed MF, Klein AP, Dong J, Maitra A, Pan D, Montgomery EA and Anders RA: Expression of Yes-associated protein in common solid tumors. Hum Pathol 39: 1582-1589, 2008

9. Xu MZ, Yao TJ, Lee NP, Ng IO, Chan YT, Zender L, Lowe SW, Poon RT and Luk JM: Yes-associated protein is an independent prognostic marker in hepatocellular carcinoma. Cancer 115: 4576-4585, 2009

10. Kapoor A, Yao W, Ying H, Hua S, Liewen A, Wang Q, Zhong Y, Wu CJ, Sadanandam A, Hu B, et al: Yapl activation enables bypass of oncogenic kras addiction in pancreatic cancer. Cell 158: 185-197, 2014.

11. Zender L, Spector MS, Xue W, Flemming P, Cordon-Cardo C, Silke J, Fan ST, Luk JM, Wigler M, Hannon GJ, et al: Identification and validation of oncogenes in liver cancer using an integrative oncogenomic approach. Cell 125: 1253-1267, 2006.

12. Lee KP, Lee JH, Kim TS, Kim TH, Park HD, Byun JS, Kim MC, Jeong WI, Calvisi DF, Kim JM and Lim DS: The Hippo-Salvador pathway restrains hepatic oval cell proliferation, liver size, and liver tumorigenesis. Proc Natl Acad Sci USA 107: 8248-8253, 2010.

13. Huang XY, Ke AW, Shi GM, Zhang X, Zhang C, Shi YH, Wang XY, Ding ZB, Xiao YS, Yan J, et al: $\alpha \mathrm{B}$-crystallin complexes with $14-3-3 \zeta$ to induce epithelial-mesenchymal transition and resistance to sorafenib in hepatocellular carcinoma. Hepatology 57 : 2235-2247, 2013.

14. Feng X, Degese MS, Iglesias-Bartolome R, Vaque JP, Molinolo AA Rodrigues M, Zaidi MR, Ksander BR, Merlino G, Sodhi A, et al: Hippo-Independent Activation of YAP by the GNAQ uveal melanoma oncogene through a trio-regulated rho GTPase signaling circuitry. Cancer Cell 25: 831-845, 2014.

15. Zhang W, Nandakumar N, Shi Y, Manzano M, Smith A, Graham G, Gupta S, Vietsch EE, Laughlin SZ, Wadhwa M, et al: Downstream of mutant KRAS, the transcription regulator YAP is essential for neoplastic progression to pancreatic ductal adenocarcinoma. Sci Signal 7: ra42, 2014.

16. Muramatsu T, Imoto I, Matsui T, Kozaki K, Haruki S, Sudol M, Shimada $\mathrm{Y}$, Tsuda $\mathrm{H}$, Kawano $\mathrm{T}$ and Inazawa J: YAP is a candidate oncogene for esophageal squamous cell carcinoma. Carcinogenesis 32: 389-398, 2011 .

17. Wang LJ, Shi SJ, Guo ZY, Zhang X, Han S, Yang A, Wen W and Zhu Q: Overexpression of YAP and TAZ Is an independent predictor of prognosis in colorectal cancer and related to the proliferation and metastasis of colon cancer cells. Plos One 8: e65539, 2013.

18. Kang W, Tong JH, Chan AW, Lee TL, Lung RW, Leung PP, So KK, Wu K, Fan D, Yu J, et al: Yes-associated protein 1 exhibits oncogenic property in gastric cancer and its nuclear accumulation associates with poor prognosis. Clin Cancer Res 17: 2130-2139, 2011.
19. Wang Y, Dong Q, Zhang Q, Li Z, Wang E and Qiu X: Overexpression of yes-associated protein contributes to progression and poor prognosis of non-small-cell lung cancer. Cancer Sci 101: 1279-1285, 2010

20. Hall CA, Wang R, Miao J, Oliva E, Shen X, Wheeler T, Hilsenbeck SG, Orsulic S and Goode S: Hippo pathway effector Yap is an ovarian cancer oncogene. Cancer Res 70: 8517-8525, 2010.

21. Zhao B, Tumaneng K and Guan KL: The Hippo pathway in organ size control, tissue regeneration and stem cell self-renewal. Nat Cell Biol 13: 877-883, 2011.

22. Wang H, Du YC, Zhou XJ, Liu H and Tang SC: The dual functions of YAP-1 to promote and inhibit cell growth in human malignancy. Cancer Metastasis Rev 33: 173-181, 2014.

23. Wang H, Zhou M, Shi B, Zhang Q, Jiang H, Sun Y, Liu J, Zhou K, Yao M, Gu J, et al: Identification of an exon 4-deletion variant of epidermal growth factor receptor with increased metastasis-promoting capacity. Neoplasia 13: 461-471, 2011.

24. Gao Q, Zhao YJ, Wang XY, Qiu SJ, Shi YH, Sun J, Yi Y, Shi JY, Shi GM, Ding ZB, et al: CXCR6 upregulation contributes to a proinflammatory tumor microenvironment that drives metastasis and poor patient outcomes in hepatocellular carcinoma. Cancer Res 72: 3546-3556, 2012.

25. Hwang JH, Pores Fernando AT, Faure N, Andrabi S, Adelmant G, Hahn WC, Marto JA, Schaffhausen BS and Roberts TM: Polyoma small $\mathrm{T}$ antigen interacts with Yes-associated protein to regulate cell survival and differentiation. J Virol 88: 12055-12064, 2014.

26. Tao J, Calvisi DF, Ranganathan S, Cigliano A, Zhou L, Singh S, Jiang L, Fan B, Terracciano L, Armeanu-Ebinger S, et al: Activation of $\beta$-catenin and Yapl in human hepatoblastoma and induction of hepatocarcinogenesis in mice. Gastroenterology 147: 690-701, 2014.

27. Fu D, Lv X, Hua G, He C, Dong J, Lele SM, Li DW, Zhai Q, Davis JS and Wang C: YAP regulates cell proliferation, migration, and steroidogenesis in adult granulosa cell tumors. Endoc Relat Cancer 21: 297-310, 2014

28. Thiery JP, Acloque H, Huang RY and Nieto MA: Epithelial-mesenchymal transitions in development and disease. Cell 139: 871-890, 2009

29. Yang J and Weinberg RA: Epithelial-mesenchymal transition: At the crossroads of development and tumor metastasis. Dev Cell 14: 818-829, 2008.

30. Acloque H, Adams MS, Fishwick K, Bronner-Fraser M and Nieto MA: Epithelial-mesenchymal transitions: The importance of changing cell state in development and disease. J Clin Invest 119: 1438-1449, 2009.

31. Zeisberg M and Neilson EG: Biomarkers for epithelial-mesenchymal transitions. J Clin Invest 119: 1429-1437, 2009.

32. Chang TH, Tsai MF, Su KY, Wu SG, Huang CP, Yu SL, Yu YL, Lan CC, Yang CH, Lin SB, et al: Slug confers resistance to the epidermal growth factor receptor tyrosine kinase inhibitor. Am J Respir Crit Care Med 183: 1071-1079, 2011.

33. Saxena M, Stephens MA, Pathak $H$ and Rangarajan A: Transcription factors that mediate epithelial-mesenchymal transition lead to multidrug resistance by upregulating $\mathrm{ABC}$ transporters. Cell Death Dis 2: e179, 2011.

34. Wang Z, Li Y, Ahmad A, Azmi AS, Kong D, Banerjee S and Sarkar FH: Targeting miRNAs involved in cancer stem cell and EMT regulation: An emerging concept in overcoming drug resistance. Drug Resist Updat 13: 109-118, 2010.

35. Nath S, Daneshvar K, Roy LD, Grover P, Kidiyoor A, Mosley L, Sahraei M and Mukherjee P: MUC1 induces drug resistance in pancreatic cancer cells via upregulation of multidrug resistance genes. Oncogenesis 2: e51, 2013.

36. Hu H, Gu Y, Qian Y, Hu B, Zhu C, Wang G and Li J: DNA-PKcs is important for Akt activation and gemcitabine resistance in PANC-1 pancreatic cancer cells. Biochem Biophys Res Commun 452: 106-111, 2014. 